\title{
Genetic Variants in Lipid Metabolism Pathways Interact with Diet to Influence Blood Lipid Concentrations in Adults with Overweight and Obesity
}

\author{
Bridget A. Hannon ${ }^{a}$ Caitlyn G. Edwards ${ }^{\text {a }}$ Sharon V. Thompson ${ }^{a}$ \\ Sarah K. Burke ${ }^{b}$ Nicholas A. Burd ${ }^{a, c}$ Hannah D. Holscher ${ }^{a, d}$ \\ Margarita Teran-Garciaa, e Naiman A. Khan ${ }^{\text {a, c }}$ \\ a Division of Nutritional Sciences, University of Illinois at Urbana-Champaign, Urbana, IL, USA; ${ }^{b}$ Department of \\ Physical Therapy, University of Florida, Gainesville, FL, USA; ' $D$ Department of Kinesiology and Community Health, \\ University of Illinois at Urbana-Champaign, Urbana, IL, USA; ${ }^{d}$ Department of Food Science and Human Nutrition,

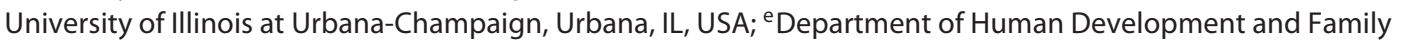 \\ Studies, University of Illinois at Urbana-Champaign, Urbana, IL, USA
}

\section{Keywords}

Lipid metabolism · Monounsaturated fat · Nutrition ·

Obesity · Nutrigenomics

\begin{abstract}
Introduction: The effect of various types of dietary fat on cardiometabolic health continues to be debated, due in part to the high heterogeneity of results following clinical trials investigating the effects of saturated (SFA) and unsaturated fat intake. This variability may be due to genetic differences. Individuals with obesity are at an increased risk for adverse cardiometabolic health and dyslipidemia, and often present with the combined phenotype of elevated triglyceride (TG) and decreased high-density lipoprotein (HDL) cholesterol concentrations. Studying genetic variants relevant to lipid and lipoprotein metabolism can elucidate the mechanisms by which diet might interact with genotype to influence these phenotypes. The objective of this study was to determine relationships of genetic variation, dietary fat intake,
\end{abstract}

karger@karger.com www.karger.com/lfg

Karger $\stackrel{\text { ' }}{5}$

BOPEN ACCESS
(C) 2020 The Author(s)

Published by S. Karger AG, Basel

This article is licensed under the Creative Commons AttributionNonCommercial-NoDerivatives 4.0 International License (CC BYNC-ND) (http://www.karger.com/Services/OpenAccessLicense) Usage and distribution for commercial purposes as well as any distribution of modified material requires written permission. and blood lipid concentrations in adults with overweight and obesity. Methods: Genomic DNA, blood lipid concentrations (HDL and TG), and 7-day diet records were obtained from 101 adults (25-45 years of age) with overweight or obesity. Resting energy expenditure (REE) was measured using indirect calorimetry and used to determine implausible intakes using a modified Goldberg method (kilocalories/REE). Genetic variants included 23 single-nucleotide polymorphisms (SNPs) from 15 genes in lipid metabolism pathways. Variants were analyzed with dietary fat intake (total fat, SFA, monounsaturated fat [MUFA], and polyunsaturated fat [PUFA]) via regression analyses. All models were adjusted for age, sex, ancestry, visceral adipose tissue mass, and total kilocalorie intake. The Bonferroni correction was applied for multiple comparisons. Results: Two interactions were detected for TG concentrations. Five gene-diet interactions were associated with HDL concentrations. There was a significant interaction detected between the rs5882 variant of cholesterol-esterase transfer protein (CETP) and MUFA intake to associate with TG concentrations (interaction $p=$ 
$\left.0.004, R^{2}=0.306\right)$. Among carriers of the CETP-rs5882 major allele (G), TG concentrations were significantly lower in individuals consuming more than the median MUFA intake (31 $\mathrm{g} /$ day) than in those with an intake below the median. Total dietary fat intake interacted with the rs 13702 polymorphism of lipoprotein lipase $(L P L)$ to associate with HDL concentrations (interaction $p=0.041, R^{2}=0.419$ ), by which individuals with the risk allele $(G)$ had significantly higher HDL concentrations when consuming a higher-fat diet ( $>92 \mathrm{~g} /$ day) than those with a lower-fat diet ( $56 \pm 3 \mathrm{vs} .46 \pm 2 \mathrm{mg} / \mathrm{dL}, p=0.033$ ). Conclusions: Interactions between dietary intake and genes in lipid metabolism pathways were found to be associated with blood lipid concentrations in adults with overweight and obesity. Fatty acid intake may not modulate blood lipid concentrations uniformly across all individuals. Additional research is needed to determine the biological causes of individual variability in response to dietary intake. Understanding the influence of nutrigenetic interactions on dyslipidemia can aid in the development and implementation of personalized dietary strategies to improve health.

\section{(c) 2020 The Author(s)}

Published by S. Karger AG, Basel

\section{Introduction}

The genetic contribution to dyslipidemia is estimated to be as high as $55 \%[1,2]$. Over 150 genetic loci have been associated with blood lipid concentrations (total cholesterol [TC], triglycerides [TG], and high-density lipoprotein [HDL] and low-density lipoprotein [LDL] cholesterol) [3]. With some notable exceptions (i.e., Tangier disease), dyslipidemia is a polygenic disease, and common variants, specifically, single-nucleotide polymorphisms (SNPs), independently have small effects on dyslipidemia. Therefore, interactions between genetic variation and environmental factors to influence dyslipidemia may explain greater variability than individual SNP effects. Dyslipidemia is the result of multiple biological and behavioral etiologies; a better understanding of these interactions can assist in development of effective strategies to prevent and treat this disease. Among the environmental factors associated with dyslipidemia, dietary intake remains one of the strongest modifiable risk factors that has been shown to interact with individual genetic variation [4]. The impact of dietary fat intake, in type and amount, on blood lipid concentrations has remained a controversial topic [5]. Saturated (SFA) and unsaturated fat (in the form of monosaturated [MUFA] and polyunsaturated [PUFA] fats) have differing metabolic consequences on the body. The results of clinical trials investigating their effects on blood lipid concentrations have been mixed; this lack of consensus regarding dietary fat intake and blood lipid concentrations may be due to genetic differences.

Examination of SNPs in the genes relevant to dietary fat and lipoprotein metabolism can potentially provide a mechanistic insight into the pathophysiology of dyslipidemia [6]. The concept that the interactions between diet and physiologically relevant genetic variants affect dyslipidemia phenotypes has been previously reviewed $[7,8]$. Dietary fat, alcohol, and adherence to a Mediterranean diet have been shown to interact with variants in cholesterol esterase transfer protein (CETP), hepatic lipase (LIPC), and glucokinase regulator protein (GCKR) to associate with blood lipid concentrations in healthy adults [9-11].

Among individuals with obesity, who are at a greater risk for metabolic abnormalities and inflammation, the independent or interactive effects of diet and genetic profile may be different. For example, the dietary replacement of SFA by unsaturated fat has been cited to be effective in the management of LDL concentrations, although this may not be as effective in adults with obesity $[12,13]$. Genotype and macronutrient composition interactively affect TC and LDL concentrations during weight loss, as demonstrated by resultsfrom the POUNDS LOST 2-year weight loss trial [14]. However, TG and HDL concentrations, which are commonly abnormal in individuals with obesity, appear to be less modifiable with gene-diet interactions [15]. Hypertriglyceridemia is strongly associated with excess visceral adipose tissue (VAT), and it promotes the exchange of TG from verylow-density lipoproteins (VLDL) for cholesterol esters to HDL particles, creating small, lipid-poor particles. As the obesity epidemic continues to rise, there is a need to understand the complex interactions underlying obesityrelated comorbidities, including dyslipidemia. The objective of this study was to investigate the interactions between dietary fat intake and lipid metabolism-related SNPs on blood lipid concentrations in adults with overweight and obesity.

\section{Materials and Methods}

\section{Participants}

Adults aged 25-45 years with overweight or obesity (body mass index $[\mathrm{BMI}] \geq 25 \mathrm{~kg} / \mathrm{m}^{2}$ ) were recruited for this cross-sectional analysis from an ongoing clinical trial (NCT02740439). Inclusion criteria involved no previous history of physician-diagnosed gastrointestinal or metabolic disease. The participants visited the lab- 
Table 1. SNPs examined in this study and previous associations with dietary fat and blood lipid concentrations

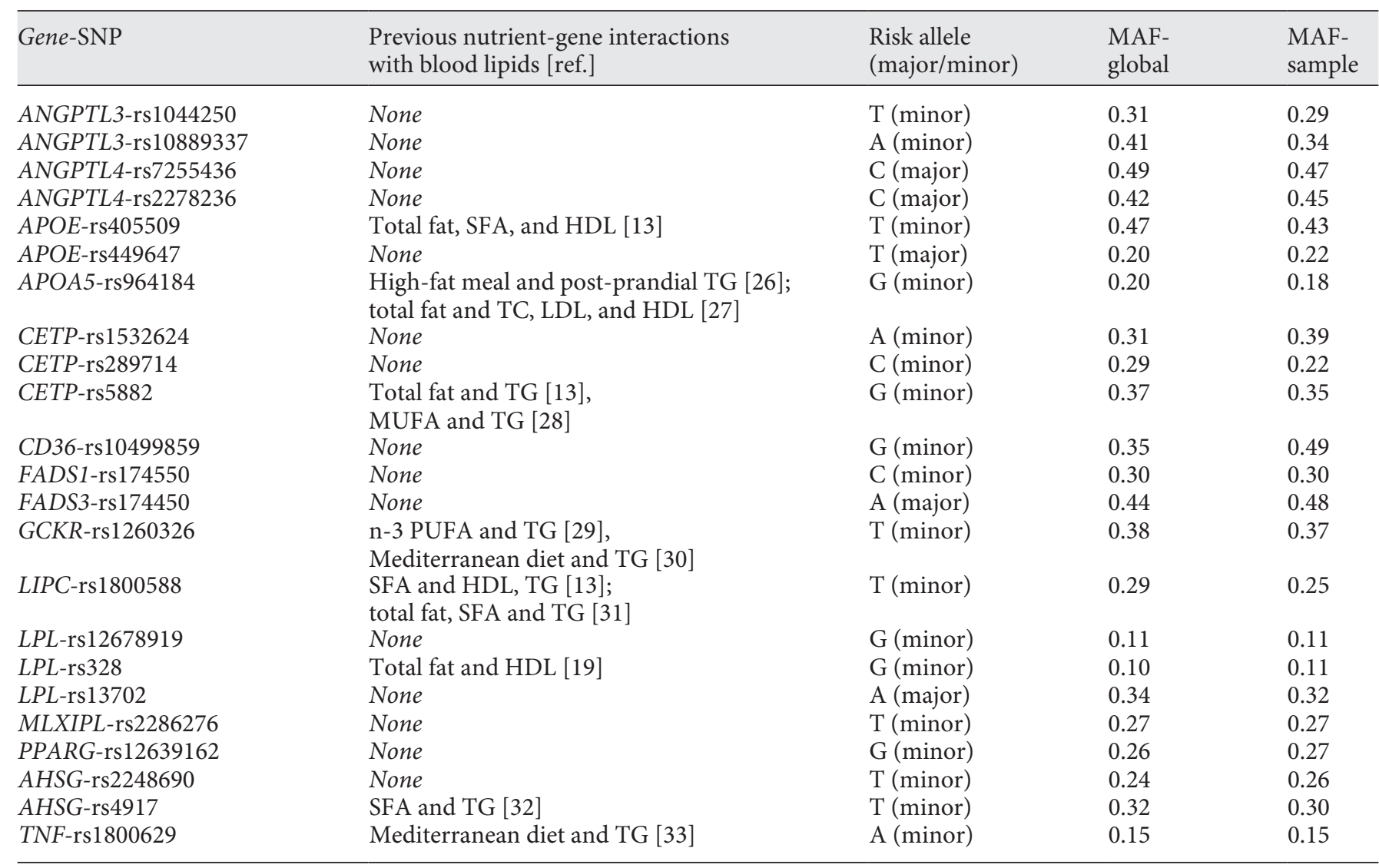

SNP, single-nucleotide polymorphism; MAF, minor allele frequency; ANGPTL3/4, angiopoietin-like protein 3/4; APOE, apolipoprotein E; APOA5, apolipoprotein A5; CETP, cholesterol esterase transfer protein; CD36, cluster of differentiation 36; FADS1/3, fatty acid desaturase 1/3; GCKR, glucokinase regulator; LIPC, hepatic lipase; LPL, lipoprotein lipase; MLXIPL, MLX interacting protein-like; PPARG, peroxisome proliferator activator receptor $\gamma$; AHSG, a Herman-Schmidt glycoprotein; TNF, tumor necrosis factor; TC, total cholesterol; LDL, low-density lipoprotein cholesterol; HDL, high-density lipoprotein cholesterol; TG, triglycerides; SFA, saturated fat; n-3 PUFA, omega-3 polyunsaturated fat.

oratory on 2 separate occasions within the same 7-day period, both after an overnight fast. The first visit consisted of a fasting blood sample for genomic and metabolic assessment. The second visit involved indirect calorimetry and a dual-energy X-ray absorptiometry (DXA) scan.

\section{Genetic Analysis and SNP Selection}

Genomic DNA was extract from whole blood using standard protocols (Gentra Puregene Blood Kit, Qiagen, Valencia, CA, USA) and genotyped at the W.M. Keck Center at the University of Illinois using the Fluidigm SNP genotyping platform. Genotypes were called on the Fluidigm Genotyping Analysis software v4.1.2 (Fluidigm Corp., San Francisco, CA, USA) with a minimum 85\% confidence. To account for the ethnic heterogeneity of the cohort, genetic samples were also genotyped for 58 ancestral informative markers [16]. From these, 49 variants (those with $>95 \%$ call rate and minor allele frequency $[\mathrm{MAF}]>10 \%)$ were included in a principal component analysis. The first 3 components accounted for $23 \%$ of the variance in the sample and were used as covariates.

Lipoprotein-Related SNPs and Dietary Fat Intake Affect Blood Lipids
From a literature review [8] and search of the NCBI database (https://www.ncbi.nlm.nih.gov/snp/), 23 SNPs in 15 genes involved in lipid and lipoprotein metabolism were identified that had been previously associated with blood lipid concentrations in humans and were functional (missense) variants, upstream transcription variants, or intronic variants previously associated with phenotypes of interest. The full list of included SNPs, previous associations with dietary fat and blood lipid concentrations, global and sample MAF is presented in Table 1. Linkage disequilibrium (LD) of SNPs in the same gene or on the same chromosome was evaluated using LDMatrix and LDLink (https://ldlink.nci.nih. gov/?tab = ldmatrix) using the CEU (Utah residents from North and Western Europe) population as a reference, as our sample was $78 \%$ Caucasian. Adherence to Hardy-Weinberg equilibrium was assessed using the $\chi^{2}$ test.

\section{Phenotype Assessment}

Blood lipid concentrations (HDL and TG) were obtained from lithium heparinized whole blood using the Piccolo Xpress Ana- 
Table 2. Demographic characteristics of study sample after the removal of implausible reporters

\begin{tabular}{|c|c|c|c|c|}
\hline & Entire sample & Males & Females & $p$ value \\
\hline$N$ & 101 & 36 & 65 & 0.08 \\
\hline Age, years & $36.2 \pm 5.8$ & $36.2 \pm 5.9$ & $36.2 \pm 5.9$ & 0.98 \\
\hline $\mathrm{BMI}, \mathrm{kg} / \mathrm{m}^{2}$ & $33.8 \pm 6.8$ & $32.8 \pm 8.3$ & $34.3 \pm 5.7$ & 0.31 \\
\hline WB \%fat, \% & $39.9 \pm 8.3$ & $32.8 \pm 8.3$ & $43.8 \pm 5.3$ & $<0.01$ \\
\hline VAT mass, $\mathrm{g}$ & $719.3 \pm 327.9$ & $730.3 \pm 380.2$ & $713.4 \pm 299.0$ & 0.81 \\
\hline $\mathrm{TC}, \mathrm{mg} / \mathrm{dL}$ & $186 \pm 32$ & $188 \pm 36$ & $185 \pm 29$ & 0.71 \\
\hline $\mathrm{TG}, \mathrm{mg} / \mathrm{dL}$ & $118 \pm 60$ & $125 \pm 67$ & $114 \pm 56$ & 0.39 \\
\hline $\mathrm{HDL}, \mathrm{mg} / \mathrm{dL}$ & $53 \pm 12$ & $48 \pm 11$ & $55 \pm 12$ & $<0.01$ \\
\hline Energy, kcal & $2,185.1 \pm 506.2$ & $2,452.2 \pm 512.8$ & $2,037.0 \pm 440.6$ & $<0.01$ \\
\hline Total fat, g & $93.9 \pm 27.4$ & $101.7 \pm 27.1$ & $89.6 \pm 26.8$ & 0.03 \\
\hline SFA, $g$ & $31.7 \pm 10.1$ & $33.1 \pm 9.6$ & $31.0 \pm 10.3$ & 0.31 \\
\hline MUFA, g & $32.9 \pm 10.1$ & $36.1 \pm 9.4$ & $31.1 \pm 10.0$ & 0.02 \\
\hline PUFA, g & $21.4 \pm 8.0$ & $23.6 \pm 9.1$ & $20.2 \pm 7.1$ & 0.04 \\
\hline
\end{tabular}

Data are presented as mean \pm SD. BMI, body mass index; WB \%fat, whole-body adipose tissue percentage; VAT, visceral adipose tissue; TC, total cholesterol; TG, triglycerides; HDL, high-density lipoprotein cholesterol; SFA, saturated fatty acids; MUFA, monounsaturated fatty acids; PUFA, polyunsaturated fatty acids. Significance between groups was determined by Student $t$ tests, with the exception of sex, which was determined by $\chi^{2}$ test.

lyzer (Abaxis, Inc., Union City, CA, USA). Height and weight were measured in triplicate by trained research staff using a Seca Model 240 stadiometer and Tanita WB-300 Plus digital scale, respectively. BMI was calculated as $\mathrm{kg} / \mathrm{m}^{2}$. VAT mass and whole-body percent fat (WB \%fat) were measured through DXA using a Hologic Horizon W bone densitometer (APEX software v5.6.0.5).

\section{Dietary Intake}

Participants were provided with a 7-day food record to be completed prior to their arrival to the laboratory. Research staff provided detailed instructions for recording food intake, and additional written instructions were contained in the record. Diet records were entered by trained research staff into the Nutrition Data Systems-Research version 2015 (NDSR, Nutrition Coordinating Center, University of Minnesota, Minneapolis, MN, USA) software. Total kilocalories (kcal), grams of total fat, SFA, MUFA, PUFA, and percentages of each of these were extracted from the NDSR output.

\section{Determination of Resting Energy Expenditure and}

Quantification of Implausible Energy Intake

Resting energy expenditure (REE) was determined via indirect calorimetry (Parvo Medics True Max 2400, Salt Lake City, UT, USA). Participants were asked to lay supine for $45 \mathrm{~min}$ with a rigid plastic canopy hood placed over their head and upper torso. Measurements of $\mathrm{O}_{2}$ usage and $\mathrm{CO}_{2}$ expiration were analyzed using a calibrated metabolic cart. REE was calculated using the Weir equation. The average REE (kcal/day) of the second 15-min segment of the testing period was used in these analyses. To determine implausible reported energy intake (EI), a modified Goldberg cutoff was applied (kcal/REE) [17]. A constant physical activity level of 1.3 was presumed for participants [18]. Individuals falling below or above 2 standard deviations (SD) of the mean were considered underreporters or overreporters, respectively.

\section{Statistical Analyses}

All variables were inspected for normality, and nonnormally distributed variables (TG) were log-transformed for analysis, and then back-transformed for interpretation. Student's $t$ tests were used to determine statistically significant differences in variables of interest by sex. Individual SNP associations were determined by general linear models (GLM) adjusted for age, sex, VAT mass, and ancestry. The genotypic, dominant, and recessive modes of inheritance were explored for SNP main effects. Type III sums of squares $p$ value was used to determine significant associations between genotype and phenotype. Main effects that were found to be significant for SNPs only were then explored with diet-SNP interaction terms. Dietary intakes of total fat, SFA, MUFA, PUFA were bifurcated by the sample median into high/low consumption to create an interaction term with genotype. GLM were then used to determine associations between blood lipid concentrations and diet-genotype interactions. These models were adjusted for age, sex, VAT mass, ancestry, total kcal intake, dietary fat intake, genotype, and the diet-genotype interaction term.

\section{Genetic Risk Score Construction}

An additive genetic risk score (GRS) was constructed from SNPs found to be individually associated with the phenotype by summing the number of risk alleles for each individual $[19,20]$. GRS were added to GLM with the aforementioned covariates first, without consideration of diet. A GRS-diet interaction term was created using the median GRS to create high/low genetic risk categories to examine potential interactions among phenotype, cumulative genetic risk, and diet. The Bonferroni correction was applied to all models to adjust for multiple comparisons. Statistical significance was set at $p<0.05$. All statistical analyses were conducted in SPSS v24 (IBM, Chicago, IL, USA). 


\section{Results}

Participants and Reports of Implausible Reported EI

A total of 112 adults provided complete data. Eleven individuals had implausible reported EI (7 underreporters and 4 overreporters) and were excluded from further analysis. Demographic characteristics for the remaining 101 individuals are presented in Table 2. Females had significantly higher WB \%fat and HDL concentrations than males. Males reported consuming higher amounts of total kcal and grams of MUFA and PUFA than females. When MUFA and PUFA were examined as percentages of total kcal, the intake was not significantly different in males and females $(p>0.05)$. Upon GLM with covariates (no genotype), only kcal intake was significantly associated with TG concentrations $(\beta=0.239, \mathrm{SE}=0.013, p=$ $0.042)$. $S e x(\beta=-7.54, \mathrm{SE}=2.61, p=0.005)$ and VAT mass $(\beta=-0.476, \mathrm{SE}=0.019, p<0.001)$ were both associated with HDL concentrations (online suppl. Table 1; see www.karger.com/doi/10.1159/000507021 for all online suppl. material).

\section{Associations of Genetic Variants and Lipid Traits}

All SNPs were found to be in Hardy-Weinberg equilibrium. Several SNPs were associated with blood lipid concentrations. The following were significantly associated with TG concentrations: CETP-rs289714, CETPrs5882, GCKR-rs1260326, LPL-rs13702, MLXIPLrs2286276. The following were significantly associated with HDL concentrations: CETP-rs289714, GCKRrs1260326, LIPC-rs1800588, LPL-rs13702. These data are presented in online supplementary Tables 2 and 3.

\section{Gene-Diet Interactions and Blood Lipid Concentrations}

Gene-diet interactions for TG and HDL concentrations are summarized in Tables 3 and 4, respectively. CETP-rs289714 significantly interacted with total fat intake to correlate with TG concentrations, by which individuals homozygous for the $\mathrm{T}$ allele (major allele) who consumed $>92 \mathrm{~g}$ total fat/day had lower TG concentrations ( $103 \pm 63 \mathrm{mg} / \mathrm{dL}$ ) than those consuming below the median $(135 \pm 15 \mathrm{mg} / \mathrm{dL} ; p=0.041)$. Both the dominant (interaction $p=0.001, R^{2}=0.294$ ) and recessive (interaction $p=0.023, R^{2}=0.208$ ) modes of inheritance models for CETP-rs289714 and total fat were significant. There was a significant interaction detected between CETP-rs5882 and MUFA intake $(p=0.004)$, by which individuals with 1 or 2 copies of the major allele $(G)$ had significantly lower TG concentrations

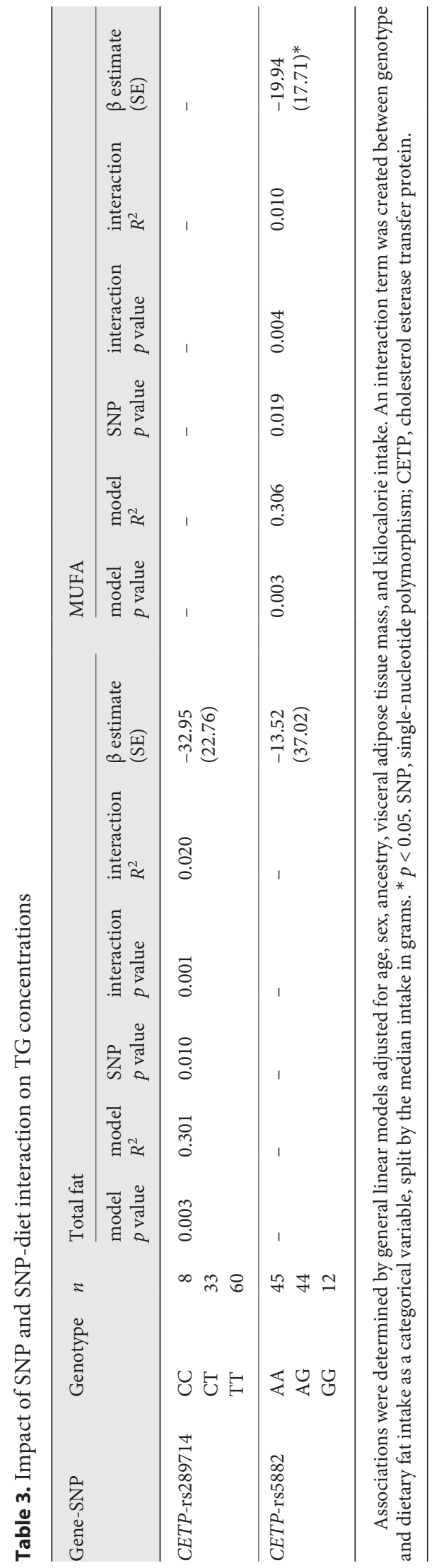




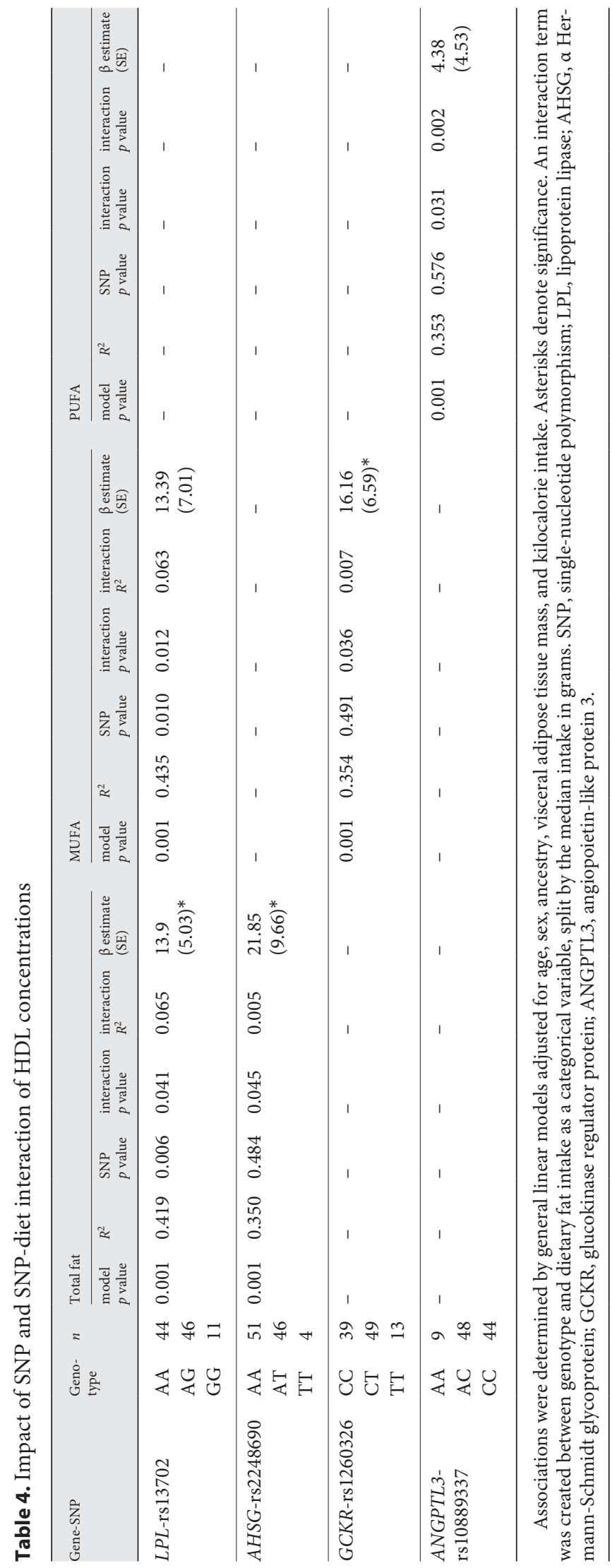

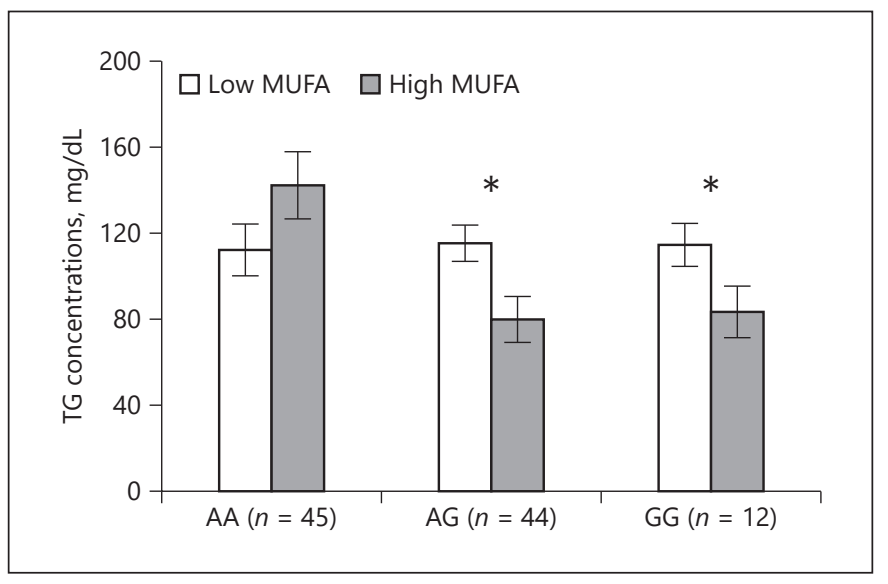

Fig. 1. Differences in TG concentrations according to CETPrs5882 genotype and MUFA intake. MUFA intake is split by the median of $31 \mathrm{~g} /$ day. Significant differences between low- and highMUFA consumers by genotype were determined by ANCOVA adjusted for age, sex, ancestry, visceral adipose tissue mass, and total kilocalorie intake. ${ }^{*} p<0.05$.

when consuming MUFA above the sample median of $31 \mathrm{~g} /$ day (Fig. 1). The recessive mode of inheritance model was statistically significant (interaction $p=0.006$, $R^{2}=0.281$ ).

Five diet-gene interactions were detected for HDL concentrations. LPL-rs 13702 significantly interacted with both total fat and MUFA intake. Individuals homozygous for the major allele (A) had higher mean HDL concentrations when consuming total fat and MUFA below the sample medians; these differences were not statistically significant $(p=0.056$ and $p=0.068$, respectively). The $L P L$-rs 13702 -total fat interaction explained $7 \%$ of variability in the model; and the $L P L$-rs 13702 MUFA interaction explained 6\%, as indicated by $R^{2}$ values. There were significant interactions for the recessive modes of inheritance with both total fat (interaction $p=$ $0.028, R^{2}=0.353$ ) and MUFA (interaction $p=0.003$, $\left.R^{2}=0.376\right)$ intake. HDL concentration means by $L P L-$ rs13702 genotype and total fat intake are depicted in Figure 2. There were significant interaction terms for AHSG-rs2248690 and total fat and GCKR-rs1260326 and MUFA, both for the genotypic modes of inheritance. The interactions between GCKR-rs 1260326 and MUFA intake was significant for the dominant mode of inheritance (interaction $p=0.019, R^{2}=0.343$ ). The interaction between ANGPTL3-rs10889337 and PUFA intake was significant for the genotypic mode of inheritance (interaction $p=0.031, R^{2}=0.353$ ). No other genediet interactions were detected. 


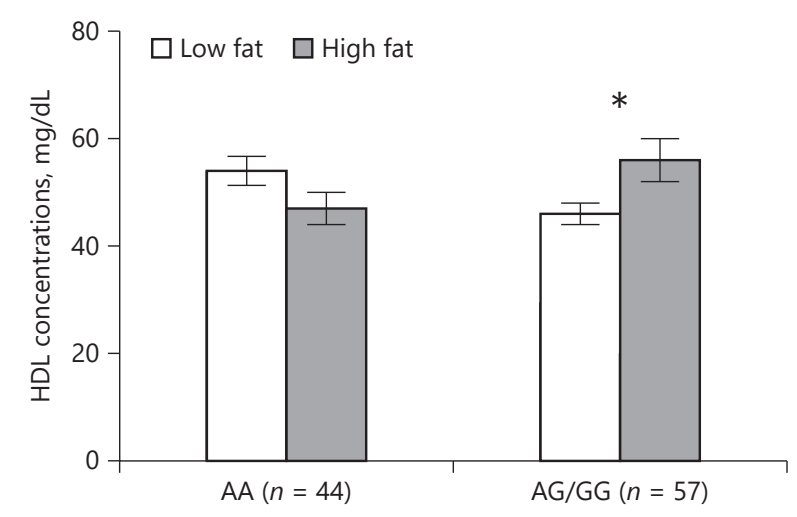

Fig. 2. Differences in HDL concentrations according to $L P L$ rs13702 genotype and total fat intake. Total fat intake is split by the median of 92 g/day. Significant differences between low- and highfat consumers by genotype were determined by ANCOVA adjusted for age, sex, ancestry, visceral adipose tissue mass, and total kilocalorie intake. ${ }^{*} p<0.05$.

\section{GRS and Lipid Traits}

The median GRS for TG and HDL was 3.0 and 2.5, respectively. The TG GRS was significantly associated with TG concentrations following adjustment for age, sex, ancestry, and VAT (Model $p=0.044 ; \beta$ estimate $=0.246$, $\mathrm{SE}=0.021$, GRS $p=0.017)$. The HDL GRS was not significantly associated with HDL concentrations $(p=0.918)$.

\section{Discussion}

This study examined SNPs in pathways of lipid and lipoprotein metabolism, enabling these findings to be physiologically relevant to dyslipidemia. There were no associations between dietary fat intake and TG or HDL concentrations, except when genotype was considered. Fatty acids differentially impact lipoprotein metabolism to influence blood lipid phenotypes, with SFA raising blood lipid concentrations through delayed clearance of apolipoprotein B-containing LDL particles, and PUFA decreasing TG concentrations through improved catabolism of apolipoprotein B-100 containing particles [21, $22]$. However, the impact of different fatty acids on lipoprotein metabolism may be altered among individuals with obesity, as this is a complex disease that impacts every organ system. For example, the replacement of SFA with unsaturated fat is recommended for the management of blood lipid concentrations, but there is no strong evidence to support its efficacy in adults with obesity [12,
13]. As the role of dietary fat in metabolic health remains controversial, investigation of diet interactions with individual factors (i.e., genetic variation) can advance the field of personalized nutrition. A similar study was conducted by Rudkowska et al. [23] in the Inuit population, and novel gene-diet interactions were detected in this understudied ethnic group. As it is known that MAF vary according to population, it is crucial to understand geneenvironment interactions different ethnic groups [24]. Additionally, as the obesity epidemic persists, there is a need to better understand the dietary and genetic determinants of comorbid diseases in this population.

The first notable gene-diet interaction was between CETP-rs5882 and MUFA intake to associate with TG concentrations. Median MUFA intake in our sample $(31.3 \mathrm{~g} /$ day) was similar to the US average reported intake of $29.6 \mathrm{~g} /$ day [25]. CETP facilitates the movement of cholesterol esters for TG in VLDL and LDL. The CETPrs5882 variant is a missense mutation that may affect the protein's ability to facilitate this exchange, potentially leading to increased TG concentrations. Fat intake may affect CETP activity in humans, as shown by De Vries et al. [26] in adults with type 1 diabetes. CETP activity was found to decrease by $12 \%$ following a 12 -week diet that decreased total fat intake to $30 \%$ of total calories but did not change MUFA intake. However, the study duration and disease state of their subjects make extrapolation of these results to other populations difficult. Esfandiar et al. [27] reported an interaction between CETP-rs5882 and MUFA intake that influenced HDL concentrations. Gallele carriers were at decreased risk for low HDL concentrations when consuming low MUFA, which is contrast to our results. There is a need for further exploration of dietary influences on the CETP-rs5882 polymorphism.

Results revealed an interaction between total fat intake and $L P L$-rs 13702 on HDL concentrations, with higher concentrations among minor allele carriers consuming $>92 \mathrm{~g} /$ day. LPL functions to hydrolyze TG in lipoproteins into free fatty acids and glycerol, the former being taken up into cells by fatty acid transport proteins. $L P L$-rs 13702 is an upstream variant and may affect transcription levels of the protein or its activity. A high-fat intake can increase LPL activity, as more substrate is provided in the form of chylomicron-derived TG [28]. LPL activity is positively associated with HDL concentrations, with the greater activity and thus catabolism of TG-rich lipoproteins resulting in elevated HDL. Diet-gene interactions with the $L P L$-rs1121923 variant and total fat intake have been detected in an Asian Indian population [29]. Similar to our study on subjects consuming a high-fat diet, risk-allele carriers had significantly higher 
HDL concentrations than noncarriers. Like rs13702, rs1121923 may affect gene expression and LPL activity.

This study had several strengths, the first being the application of Goldberg cutoffs to remove individuals reporting implausible EI. Self-report methods of EI can be unreliable due to recall bias, misestimation of portion sizes, or social desirability bias $[30,31]$. Although there are reports that individuals are better able to recall what types of foods they ate rather than how much, there is still a need to identify and exclude implausible reporters to prevent inaccurate conclusions on diet-disease relationships [32]. Among adults with obesity, underreporting is more commonly observed, but overreporting also occurs and this must be addressed [31]. The Goldberg cutoffs utilize the ratio of estimated energy expenditure (using formulae such as Mifflin-St. Jeor or Schofield) to reported EI to exclude individuals reporting EI outside of physiologically plausible ranges [17]. Another strength was the study population. Young-to-middle aged adults, such as those included in our study, have a high prevalence of obesity (36\% for the age group $20-39$ years and $43 \%$ for $40-59$ years), but they are understudied with regard to diet-disease relationships [33]. Despite having overweight and obesity, these adults are currently free from chronic disease, a state that may be temporary but can potentially be prolonged through lifestyle management [34].

This study did have several limitations. Physical activity levels were not able to be assessed from the available diet records. Additionally, a sample size of 101 is small compared to other genetic association studies. However, the adjustment for multiple testing comparisons and the selection of biologically relevant variants mitigated the potential for spurious associations. Additionally, through the inclusion of ancestral informative markers and adjustment for ancestry, different ethnicities were able to be analyzed together, rather than selecting only one population. Most genetic research has been conducted in individuals of European descent, and there is a need for greater research diversity [35].
In conclusion, this study identified gene-diet associations between lipoprotein metabolism-related SNPs and dietary fat intake on blood lipid concentrations among adults with overweight and obesity. Overweight and obesity are conditions that predispose afflicted individuals to numerous comorbidities, including dyslipidemia. Understanding the modifiable and nonmodifiable factors that contribute to this phenotype can inform future dietary approaches for prevention or treatment.

\section{Statement of Ethics}

All study procedures were approved by the University of Illinois Institutional Review Board. Participants provided written informed consent prior to any procedures.

\section{Conflict of Interest Statement}

All authors have no conflicts of interest to disclose.

\section{Funding Sources}

This work was supported by the Hass Avocado Board, the Department of Kinesiology and Community Health at the University of Illinois and the USDA National Institute of Food and Agriculture, Hatch Project 1009249. BAH and MTG are supported by the Agriculture and Food Research Initiative Competitive Grant no. 2015-68001-23248 from the USDA National Institute of Food and Agriculture (NIFA) to Cooperative Extension and the Department of Human Development and Family Studies. SVT was supported by the University of Illinois College of ACES Jonathan Baldwin Turner Fellowship.

\section{Author Contributions}

B.A.H.: analyzed data and wrote the manuscript. N.A.B., H.D.H., and N.A.K.: conceptualized the study. B.A.H., S.K.B., C.G.E., and S.V.T.: collected data. M.T.G. and N.A.K.: edited the manuscript. All authors approved the final content of the manuscript.

\section{References}

1 Watanabe K, Stringer S, Frei O, Umićević Mirkov M, de Leeuw C, Polderman TJ, et al. A global overview of pleiotropy and genetic architecture in complex traits. Nat Genet. 2019 Sep;51(9):1339-48.

2 Willer CJ, Schmidt EM, Sengupta S, Peloso GM, Gustafsson S, Kanoni S, et al.; Global Lipids Genetics Consortium. Discovery and refinement of loci associated with lipid levels. Nat Genet. 2013 Nov;45(11):1274-83.
3 Weissglas-Volkov D, Pajukanta P. Genetic causes of high and low serum HDL-cholesterol. J Lipid Res. 2010 Aug;51(8):2032-57.

4 Cole CB, Nikpay M, McPherson R. Gene-environment interaction in dyslipidemia. Curr Opin Lipidol. 2015 Apr;26(2):133-8.

5 Lichtenstein AH. Dietary Fat and Cardiovascular Disease: Ebb and Flow Over the Last Half Century. Adv Nutr. 2019 Nov; 10 Suppl_4:S332-9.
6 Paynter NP, Chasman DI, Paré G, Buring JE Cook NR, Miletich JP, et al. Association between a literature-based genetic risk score and cardiovascular events in women. JAMA. 2010 Feb;303(7):631-7.

7 Abdullah MM, Jones PJ, Eck PK. Nutrigenetics of cholesterol metabolism: observational and dietary intervention studies in the postgenomic era. Nutr Rev. 2015 Aug;73(8):523-43. 
8 Hannon BA, Khan NA, Teran-Garcia M. Nutrigenetic contributions to dyslipidemia: A focus on physiologically relevant pathways of lipid and lipoprotein metabolism. Nutrients. 2018 Oct;10(10):10.

9 Mirmiran P, Esfandiar Z, Hosseini-Esfahani F, Koochakpoor G, Daneshpour MS, Sedaghati-Khayat B, et al. Genetic variations of cholesteryl ester transfer protein and diet interactions in relation to lipid profiles and coronary heart disease: a systematic review. Nutr Metab (Lond). 2017 Dec;14(1):77.

10 Ordovas JM, Corella D, Demissie S, Cupples LA, Couture P, Coltell O, et al. Dietary fat intake determines the effect of a common polymorphism in the hepatic lipase gene promoter on high-density lipoprotein metabolism: evidence of a strong dose effect in this genenutrient interaction in the Framingham Study. Circulation. 2002 Oct;106(18):231521.

11 Sotos-Prieto M, Guillén M, Vicente Sorli J, Portolés O, Guillem-Saiz P, Ignacio Gonzalez J, et al. Relevant associations of the glucokinase regulatory protein/glucokinase gene variation with TAG concentrations in a highcardiovascular risk population: modulation by the Mediterranean diet. Br J Nutr. 2013 Jan;109(2):193-201.

12 Hannon BA, Thompson SV, An R, TeranGarcia M. Clinical outcomes of dietary replacement of saturated fatty acids with unsaturated fat sources in adults with overweight and obesity: A systematic review and metaanalysis of randomized control trials. Ann Nutr Metab. 2017;71(1-2):107-17.

13 Sacks FM, Lichtenstein AH, Wu JH, Appel LJ, Creager MA, Kris-Etherton PM, et al.; American Heart Association. Dietary fats and cardiovascular disease: A presidential advisory from the American heart association. Circulation. 2017 Jul;136(3):e1-23.

14 Zhang X, Qi Q, Bray GA, Hu FB, Sacks FM, Qi L. APOA5 genotype modulates 2-y changes in lipid profile in response to weight-loss diet intervention: the Pounds Lost Trial. Am J Clin Nutr. 2012 Oct;96(4):917-22.

15 Després JP, Lemieux I. Abdominal obesity and metabolic syndrome. Nature. 2006 Dec; 444(7121):881-7.

16 Kosoy R, Nassir R, Tian C, White PA, Butler LM, Silva G, et al. Ancestry informative marker sets for determining continental origin and admixture proportions in common populations in America. Hum Mutat. 2009 Jan;30(1): 69-78.

17 Goldberg GR, Black AE, Jebb SA, Cole TJ, Murgatroyd PR, Coward WA, et al. Critical evaluation of energy intake data using fundamental principles of energy physiology: 1 . Derivation of cut-off limits to identify underrecording. Eur J Clin Nutr. 1991 Dec;45(12): 569-81.

18 Tooze JA, Krebs-Smith SM, Troiano RP, Subar AF. The accuracy of the Goldberg method for classifying misreporters of energy intake on a food frequency questionnaire and 24-h recalls: comparison with doubly labeled water. Eur J Clin Nutr. 2012 May;66(5):569-76.

19 Guevara-Cruz M, Medina-Vera I, FloresLópez A, Aguilar-López M, Smith CE, Parnell LD, et al. Development of a Genetic Score to Predict an Increase in HDL Cholesterol Concentration After a Dietary Intervention in Adults with Metabolic Syndrome. J Nutr. 2019 Jul;149(7):1116-21.

20 Wang AA, Harrison K, Musaad S, Donovan SM, Teran-Garcia M; STRONG Kids Research Team. Genetic risk scores demonstrate the cumulative association of single nucleotide polymorphisms in gut microbiome-related genes with obesity phenotypes in preschool age children. Pediatr Obes. 2019 Sep; 14(9):e12530.

21 Lamarche B, Couture P. Dietary fatty acids, dietary patterns, and lipoprotein metabolism. Curr Opin Lipidol. 2015 Feb;26(1):42-7.

22 Skulas-Ray AC, Wilson PW, Harris WS, Brinton EA, Kris-Etherton PM, Richter CK, et al.; American Heart Association Council on Arteriosclerosis, Thrombosis and Vascular Biology; Council on Lifestyle and Cardiometabolic Health; Council on Cardiovascular Disease in the Young; Council on Cardiovascular and Stroke Nursing; and Council on Clinical Cardiology. Omega-3 fatty acids for the management of hypertriglyceridemia: A science advisory from the American Heart Association. Circulation. 2019 Sep;140(12):e673-91.

23 Rudkowska I, Dewailly E, Hegele RA, Boiteau V, Dubé-Linteau A, Abdous B, et al. Genediet interactions on plasma lipid levels in the Inuit population. Br J Nutr. 2013 Mar; 109(5): 953-61.

24 Vazquez-Vidal I, Voruganti VS, Hannon BA, Andrade FC, Aradillas-Garcia C, Nakamura MT, et al. Serum lipid concentrations and FADS genetic variants in young Mexican college students: The UP-AMIGOS cohort study. Lifes Genom; 2018. pp. 1-9.

25 U.S. Department of Agriculture ARS, Beltsville Human Nutrition Research Center, Food Surveys Research Group. (Beltsville, MD) and U.S. Department of Health and Human Services, Centers for Disease Control and Prevention. What We Eat in America, NHANES
2015-2016 Data: Energy Intakes, Individuals 20 years and over, Day 1. (June, 2019). National Center for Health Statistics (Hyattsville, MD); 2017 [07/11/2019.]. Available from: https://www.ars.usda.gov/ARSUserFiles/80400530/pdf/1516/Table_5_EIN_ GEN_15.pdf.

26 De Vries R, Beusekamp BJ, Kerstens MN, Groen AK, Van Tol A, Dullaart RP. A lowsaturated-fat, low-cholesterol diet decreases plasma CETP activity and pre $\beta$-HDL formation but does not affect cellular cholesterol efflux to plasma from type 1 diabetic patients. Scand J Clin Lab Invest. 2005;65(8):729-37.

27 Esfandiar Z, Hosseini-Esfahani F, Daneshpour MS, Zand H, Mirmiran P, Azizi F. Cholesteryl ester transfer protein gene variations and macronutrient intakes interaction in relation to metabolic syndrome: Tehran lipid and glucose study. Iran J Basic Med Sci. 2018 Jun; 21(6):586-92.

28 Kiens B, Essen-Gustavsson B, Gad P, Lithell $\mathrm{H}$. Lipoprotein lipase activity and intramuscular triglyceride stores after long-term high-fat and high-carbohydrate diets in physically trained men. Clin Physiol. 1987 Feb;7(1):1-9.

29 Ayyappa KA, Shatwan I, Bodhini D, Bramwell LR, Ramya K, Sudha V, et al. High fat diet modifies the association of lipoprotein lipase gene polymorphism with high density lipoprotein cholesterol in an Asian Indian population. Nutr Metab (Lond). 2017 Jan; 14(1): 8-8.

30 Heymsfield SB, Darby PC, Muhlheim LS, Gallagher D, Wolper C, Allison DB. The calorie: myth, measurement, and reality. Am J Clin Nutr. 1995 Nov;62(5 Suppl):1034S-41S.

31 Schoeller DA, Bandini LG, Dietz WH. Inaccuracies in self-reported intake identified by comparison with the doubly labelled water method. Can J Physiol Pharmacol. 1990 Jul; 68(7):941-9.

32 Livingstone MB, Black AE. Markers of the validity of reported energy intake. J Nutr. 2003 Mar;133 Suppl 3:895S-920S

33 Division of Nutrition Physical Activity and Obesity. National Center for Chronic Disease Prevention and Health Promotion. Adult Obesity Facts Washington, D.C.2018 [07/13/2019]. Available from: https://www. cdc.gov/obesity/data/adult.html.

34 Magkos F. Metabolically healthy obesity: what's in a name? Am J Clin Nutr. 2019 Sep; 110(3):533-9.

35 Bustamante CD, Burchard EG, De la Vega FM. Genomics for the world. Nature. 2011 Jul;475(7355):163-5. 